\title{
Interactive comment on "Sulphur mass balance and radiative forcing estimation for a moderate volcanic eruption using new sulphate aerosols retrievals based on IASI observations" by H. Guermazi et al.
}

\section{Anonymous Referee \#1}

Received and published: 30 October 2019

The manuscript by Guermazi et al., deals with the retrieval of sulphate aerosols from observations of the IASI satellite sounder of the 18 March 2012 Etna eruption. Vertical profiles are retrieved and the results are discussed and compared together with the corresponding SO2 retrievals. Finally an estimate is giving of the direct radiative forcing due to the aerosols.

I am sorry that I cannot be more positive, but even from a cursory read during the technical review, it is/was obvious that the manuscript was not ready for peer review as it misses several crucial elements, that not only make the science non-reproducible, 
but that prevent the reader from judging whether the paper is scientifically sound. As further detailed below, the manuscript in its current state, is indeed very far from being publishable. The missing elements need to be added to the manuscript, and after that it needs to be completely re-reviewed.

Major comments:

Interactive

comment

\section{Spectroscopic evidence}

Very strong conclusions are drawn from the retrieval results. However, the interpretation of the results is premature, and the authors should first provide evidence that what is retrieved are actually sulfate aerosols. For this I suggest:

- a figure with an IASI observed spectrum (two spectra ideally, one in the high and one in the low altitude part), together with the spectral fit, with and without accounting for sulphates, and which show clearly the sulfate signature in the observed spectra (and with a magnitude that is consistent with the retrieved concentration). This plot should also show that a good fit cannot be obtained when sulfates are not included when both SO2 and volcanic ash is accounted for (see also next point)

- it is completely unclear how $\mathrm{SO} 2$, volcanic ash and clouds may interfere with the retrieval, which all absorb in the same spectral range, and whether AEROIASI is able to disentangle the three. Which of those are taken into account? How do clouds, volcanic ash and $\mathrm{SO} 2$ affect the retrieval?

- an analysis of the spectra outside the volcanic plume where the sulfate mass is nonzero. Rather than speculating on the origin of these enhancements, an analysis of these spectra needs to be carried out. If we cannot trust the retrieval outside the volcanic plume, why would we trust it inside?

Printer-friendly version

- in addition to this, the "geographically consistency" that the authors claim to see between SO2 and the sulfates is far from convincing. Comparing by hand the individual pixels is in any case not the proper way of doing this. For this I would suggest a 
colorcoded plot, where all IASI pixels are shown, and where each pixel is color coded ( 4 colors) along the lines of:

1. no SO2 or sulfates

2. SO2 alone

Interactive

3. sulfates alone

comment

4. both SO2 and sulfates detected

In addition, the pixels could have a different symbol depending on whether the pixels contains meteorological clouds or not. Such a figure would allow proper discussion of the plume and the retrieval.

\section{Retrieval}

As pointed out in detail by the other reviewer, the description of the retrieval is not nearly detailed enough. In addition to the very good questions and comments the other reviewer makes, the following need to be addressed:

- justification of the size distribution. From the text it seems that these are based on a sensitivity study (so what IASI can see), rather than on what can be expected physically. The width of the distribution in particular is bound to have a large impact on the retrieved masses, and needs to be justified carefully

- error budget: the $15 \%$ uncertainty is completely unrealistic. It is clear that the main uncertainties are not taken into account (width of the size distribution, spectral interferences, altitude). With respect to altitude: the retrieved profile (a $7 \mathrm{~km}$ thick layer in case of the high plume), is not a realistic profile, as the actual plume would be very narrow vertically $(\sim 1 \mathrm{~km})$. Not only does it mean that the retrieved sulphate mass will have an error of $100 \%$ at all the other altitudes, it also means that the sulphate mass at the actual height of the plume will be largely underestimated. In addition, having a profile this far from reality, will also mean that large errors are introduced on the total column, 
due to the fact that the sensitivity of the sulfate aerosols increases with altitude (in other words, if the retrievals were to be redone with the assumption of a narrow profile that peaks at the altitude where the current vertical profile peaks, the results would be very different).

- why is a vertical profile retrieved when the information content is only 1 degree of freedom on the altitude? Would it be not much better to assume a fixed layer thickness and retrieve the center altitude of this layer? (from what I understand, this is how the $\mathrm{SO} 2$ altitude is retrieved)

- what assumptions are made on the spectrally varying surface emissivity?

- as mentioned before: the authors need to explain how clouds, SO2 and volcanic ash are taken into account in the retrieval. In this way also, a value of $\mathrm{SO} 2$ will be obtained that be compared with the independently retrieved "Oxford" SO2? Vice verse: what is the impact of the sulfate aerosols on the Oxford SO2?

- the $57 \% \mathrm{H} 2 \mathrm{SO} 4$ mixing ratio (I guess this refers to the refractive index data that was used) needs to be justified.

- there is a dependence of the refractive index data on temperature in the Biermann data, has this been taken into account? If so, for each altitude layer, a different refractive index need to be used. Please detail and justify.

- why was the ERA-Interim data used for the surface temperature and water vapor profiles? I recommend using the much improved ERA5 dataset. What was the input data for the vertical temperature profiles?

- Please provide details on the R matrix other than "constant"? What was the constant?

- Please provide a plot of the cloud cover for the analyzed scene(s) (possibly to be added to the color-coded plot as requested above)

- Please provide a plot of the DOFS in and outside the plume. Note that the DOF

Interactive

comment

Printer-friendly version

Discussion paper 
outside of 0.81 seems extremely high, and again calls into question the reliability of the retrieval.

3. Analysis of the plume before and after the 19 March daytime overpass

Extremely bold statements are made in lines 173-185, which are based on the assumption that the retrieval is correct (and thus on the overly optimistic error budget). What would make this analysis (and in fact the entire paper) convincing is an analysis of the SO2 and sulfate burdens 12 hours before and 12 hours after the 19 March daytime orbit. According to the IASI data displayed on http://sacs.aeronomie.be, the different plumes are readily detectable on those overpasses (and indeed as the authors point out, the plume that is shown is more than 24 hours after the eruption started). It is puzzling that the authors do not show their retrieval on these plumes (especially on the first overpass - what would the justification be not to show it?). Having three data points would allow to estimate SO2 lifetime, and SO2 to sulphate conversion rates, and if consistency is found, would convincingly demonstrate the skill of the retrieval. As it stands, all the interpretation on the sulphur mass balance is speculation at best. In addition, the three data points would enable the authors to make a first realistic estimate of the uncertainty of their retrievals (at least on the random error). Addressing this third point, is in my opinion equally important as the previous two points.

4. general comment on the tone of the manuscript

In general the manuscript is well written, but much interpretation and main conclusions (as stated in the abstract) are stated as facts, and these should be formulated much more carefully, especially when so little (or even contradictory) evidence is provided.

Minor comments:

Line 127: in the south of -> to the south of

Printer-friendly version

Line 143: the correct past tense of split is split, not splitted

Discussion paper

Line 190: "Even if", what does this refer to?

Interactive comment 
Line 197: what does "ashy" mean? mineral ash?

Line 203: the fact that was -> the fact that this was

AMTD

Line 224: chimere -> CHIMERE

Interactive comment on Atmos. Meas. Tech. Discuss., doi:10.5194/amt-2019-341, 2019.

Interactive comment 